\title{
Modelling Consumer Behavior by Inverse Demand Functions
}

\author{
Susanne Fuchs-Seliger \\ Institut für Volkswirtschaftslehre Karlsruher Institut für Technologie, Karlsruher, Deutschland \\ Email: susanne.fuchs-seliger@kit.edu
}

Received June 4, 2013; revised July 4, 2013; accepted July 18, 2013

Copyright (C) 2013 Susanne Fuchs-Seliger. This is an open access article distributed under the Creative Commons Attribution License, which permits unrestricted use, distribution, and reproduction in any medium, provided the original work is properly cited.

\begin{abstract}
In this article a model of consumer behavior will be developed, based on preferences on the price space reflecting the individual's willingness to pay for certain quantities of commodities under the supposition that the individual is restricted to his or her income. Firms offer certain amounts of commodities at the market and consumers react to these offers by their willingness to pay. Existence and continuity of the inverse demand function describing consumer's behavior under appropriate conditions will be shown. Furthermore, differences between a model of consumer behavior based on preferences on the commodity space and that which is based on preferences on the price space will be pointed out.
\end{abstract}

Keywords: Consumer Theory; Preference Relation; Inverse Demand Functions; Duality Theory

\section{Introduction}

We will consider inverse demand functions which assign, to every commodity bundle $x$, those market price combinations $p$ the individual is willing to pay for $x$ at most when income $M$ prevails. The demand function, defined on prices and income, and the related inverse demand function, defined on commodities offered at the market and on the individual's income, are dual concepts describing consumer behavior. According to its definition, a demand function associates with every budget set $B(p, M)=\{x \in X \mid p x \leq M\}$, that commodity bundle which according to the preferences of the individual is the one he or she prefers to the other ones available in the given budget set. From the point of duality, according to the individual's preferences with regard to prices, the inverse demand function points out that price combination the individual would spend for $x$ at most, given income $M$. As an example we may consider the following one: the individual is not willing to pay more than two Euro for one pound of bred. Then he can afford to buy cheese for not more than three Euro and ham for two Euro. In total the individual cannot spend more than seven Euro for these goods. Evidently, he would be happy if these commodities were cheaper. As another example we can fancy a market where carpets are sold and the agent is not willing to pay more than a certain amount of money for a special carpet.
Inverse demand functions often are a convenient tool for modelling market behavior in the presence of monopolistic firms ([1], p. 326). If the firms have information about the individual's preferences on prices, then they will know that he or she cannot accept prices higher than a certain limit.

The analysis in this article will be based on preference relations on prices instead of indirect utility functions. Therefore, a more general framework for inverse demand will be established. We will introduce axioms concerning consumer's preferences on the price space $\mathrm{IR}_{++}^{n}$. Based on these axioms properties of the inverse demand function will be deduced. For comparison, we will also point out the difference between a model of consumer behavior based on preferences on the commodity space and that which is based on the price space.

\section{Hypotheses on Consumer's Preferences}

Modelling consumer's behavior we will assume the following hypotheses:

(P1) $\succeq$ is a relation on the strictly positive n-dimensional vector space $\mathrm{IR}_{++}^{n}$. Every $p \in \mathrm{IR}_{++}^{n}$ represents an income-normalized price vector, i.e. $p=\frac{P}{M}$, where $P \in \mathbb{R}_{++}^{n}$ are the market prices, $P_{i}$ is the price of one unit of the good $x_{i}$, and $M$ is the income of the individual, where $M>0$. 
(P2) $\succeq$ is transitive and complete ${ }^{1}$.

$(\mathrm{P} 3) \succeq$ is continuous, i.e.

(P3.1) $\left\{p \in \operatorname{IR}_{++}^{n} \mid p^{0} \succeq p\right\}$ is closed in $\operatorname{IR}_{++}^{n}$ for every $p^{0} \in \mathrm{IR}_{++}^{n}$ (lower semicontinuity),

(P3.2) $\left\{p \in \mathrm{IR}_{++}^{n} \mid p \succeq p^{0}\right\}$ is closed in $\operatorname{IR}_{++}^{n}$ for every $p^{0} \in \mathrm{IR}_{++}^{n} \quad$ (upper semicontinuity).

(P4) $p^{1}<p^{2} \Rightarrow p^{1} \succ p^{2}$, where $\succ$ is the asymmetric part of $\succeq^{2}$.

(P5) Let $\left\langle p^{\bar{k}}\right\rangle, p^{k} \in \mathrm{IR}_{++}^{n}$, be a sequence such that $\lim _{k \rightarrow \infty} p^{k}=p^{0} \ngtr 0$, where $0 \in \mathrm{IR}_{+}^{n}$, and $p \in \operatorname{IR}_{++}^{n}$. Then there exists a positive integer $N$ such that $p^{k} \succ p$ for all $k>N$.

The term $p \succeq p^{\prime}$ means: the consumer either prefers the price vector $p$ to $p^{\prime}$ or he is indifferent between them. (P4) should be interpreted as follows: if the individual can achieve a commodity bundle $x$ in the price situation $p^{1}$ at which all of the commodity prices are lower than those in the price situation $p^{2}$, then the individual prefers $p^{1}$ to $p^{2}$.

(P5) can be interpreted as: if the prices of some goods turn to 0 , then the value of the whole price systems increases and becomes greater than any given positive price system $p$. However, the value may not converge to infinity, but to a point of saturation. (P5) is a regularity condition for proving Theorem 1 and 3 . In reality no market price turns to 0 .

\section{Inverse Demand Functions}

Let us consider a commodity space $X \subseteq \mathrm{IR}_{+}^{n}$ and the price space $\mathrm{IR}_{++}^{n}$. Then the mapping $b: X \rightarrow 2^{\mathrm{IR}_{++}^{n}}, b(x) \neq \varnothing$, is called an "inverse demand correspondence', If $b(x)$ is single-valued, then $b$ is called an inverse demand function. $2^{\mathrm{IR}_{++}^{n}}$ denotes the power set of $\mathrm{IR}_{++}^{n}$.

By $B(x)$ we will denote all those income-normalized price vectors at which $x$ is available, i.e.

$B(x)=\left\{p \in \operatorname{IR}_{++}^{n} \mid p x \leq 1\right\}$. It has been shown (see [2], Theorem 1, pp. 241-242), that the correspondence $B: \operatorname{IR}_{+}^{n} \rightarrow 2^{\mathbb{I R}_{++}^{n}}, B(x)=\left\{p \in \operatorname{IR}_{++}^{n} \mid p x \leq 1\right\}$, is lower hemicontinuous.

The inverse demand function corresponds to the indirect utility function being the dual counterpart to the (direct) utility function to which the demand function corresponds [3]. Given an indirect utility function $v$ or, more generally, a preference relation $\succeq$ on the (income-normalized) price space $\mathrm{IR}_{++}^{n}$, we will ask when the equality

\footnotetext{
${ }^{1} \forall p, p^{\prime} \in \mathrm{IR}_{++}^{n}: p \succeq p^{\prime} \vee p^{\prime} \succeq p$.

${ }^{2} p^{1}<p^{2}$ means, $p_{i}^{1}<p_{i}^{2} \forall i \leq n$.
}

$$
\left\{p \in \mathrm{IR}_{++}^{n} \mid p \in B(x) \wedge \forall p^{\prime} \in B(x): p^{\prime} \succeq p\right\} \neq \varnothing,
$$

holds. Then we can define an inverse demand correspondence $b: X \rightarrow 2^{\mathrm{IR}_{++}^{n}}$ such that $b(x)=\left\{p \in \operatorname{IR}_{++}^{n} \mid p \in B(x) \wedge \forall p^{\prime} \in B(x): p^{\prime} \succeq p\right\}$. Thus, $b(x)$ points out special real price vectors $P \in \mathrm{IR}_{++}^{n}$ at income $M$ of those price vectors, which are at the individual's disposal so that $P x \leq M$, i.e. $p x=\frac{P}{M} x \leq 1$, holds (for $M>0$ ) when commodity bundle $x$ is available at the market.

By interpretation, $b(x)$ represents the set of all those income-normalized price vectors which are most unfavourable in the budget set $B(x)$ according to the individual's opinion. Therefore, producers of the commodity bundle $x$ should know that the individual will not accept prices still worse than those indicated by $b(x)$. In case the profit maximizing firms are content with those prices, a market equilibrium can be attained. In view of (P4) $b(x)$ consists of the highest prices the individual is willing to pay at most for $x$ under the restriction that he or she is limitated by his or her income.

If $b(x)$ satisfies equality (1), then we will also call $b: X \rightarrow 2^{\mathrm{IR}_{++}^{n}}$ as to be "consistent with $\succeq$ ". The above definition of consistency can be considered as the dual counterpart to rationality of demand correspondences with respect to a given relation $R$ on the commodity space $X \subseteq \mathrm{IR}_{+}^{n}$, i.e.:

$$
h(p)=\left\{x \in X \mid x \in B^{\prime}(p) \wedge \forall y \in B^{\prime}(p): x R y\right\},
$$

where

$$
B^{\prime}(p)=\{z \in X \mid p z \leq 1\}, \forall p \in \operatorname{IR}_{++}^{n} .
$$

For comparison, if we start describing consumer behavior based on preferences on the commodity space, then one can impose the following hypotheses (A1) to (A4) on the commodity space $X$, and on the relation $R$ on $X$ :

(A1) $X \subseteq \mathrm{IR}_{+}^{n}, X \neq \varnothing$, is supposed to be a closed set of alternatives.

(A2) $R$ is a reflexive relation on $X$.

(A3) $R$ is upper semicontinuous on $X$, i.e. $R(x)=\{y \mid y \in X \wedge y R x\}$ is closed in $X$ for all $x \in X$.

(A4) For all $z \in X, \tilde{P}(z)=\{y \mid y \in X \wedge \neg(z R y)\}$ is supposed to be convex and non-empty.

Then $h(p)=\left\{x \mid x \in B^{\prime}(p) \wedge \forall y \in B^{\prime}(p): x R y\right\} \neq \varnothing$ (see [4], p. 303).

We can realize that these hypotheses are quite mild. Even transitivity and completeness of the individual's preferences on the commodity space are not assumed. The proof has been done by the help of the finite intersection property since the budget sets $B^{\prime}(p)=\{x \in X \mid p x \leq 1\}, \forall p \in \mathrm{IR}_{++}^{n}$, are compact. How- 
ever, since $B(x)=\left\{p \in \mathbb{I R}_{++}^{n} \mid p x \leq 1\right\}$ is not compact, the finite intersection property cannot be applied for showing $b(x) \neq \varnothing$, and we therefore need different assumptions.

If we assume (P1) to (P5), then $b(x) \neq \varnothing$ follows. This will be shown by the next theorem.

Theorem 1 Let the commodity space be the $\mathrm{IR}_{++}^{n}$ and assume (P1) to (P5). Then for all $x \in \mathrm{IR}_{++}^{n}$, $b(x)=\left\{p \in \mathbb{I R}_{++}^{n} \mid p \in B(x) \wedge \forall p^{\prime} \in B(x): p^{\prime} \succeq p\right\} \neq \varnothing$.

\section{Proof.}

By assumption $\succeq$ is a complete, transitive and continuous relation on $\mathrm{IR}_{++}^{n}$. Therefore, by Debreu's representation theorem [5], it can be represented by a continuous function $v: \mathrm{IR}_{++}^{n} \rightarrow \mathrm{IR}_{+}$such that $p \succeq p^{\prime} \Leftrightarrow v(p) \geq v\left(p^{\prime}\right), \forall p, p^{\prime} \in \mathbb{I}_{++}^{n}$. The function $v$ will be interpreted as an indirect utility function. In view of (P4) $v$ is decreasing ${ }^{3}$. It suffices to show now, that for all $x \in \mathrm{IR}_{++}^{n}$,

$$
\begin{aligned}
& b(x) \\
& =\left\{p \in \mathbb{I R}_{++}^{n} \mid p \in B(x) \wedge \forall p^{\prime} \in B(x): v\left(p^{\prime}\right) \geq v(p)\right\} \\
& \neq \varnothing .
\end{aligned}
$$

Since $v(p)$ is decreasing

$$
\begin{aligned}
& \inf \left\{v(p) \mid p x=1, p \in \mathbb{I}_{++}^{n}\right\} \\
& =\inf \left\{v(p) \mid p x \leq 1, p \in \mathbb{I}_{++}^{n}\right\} \\
& \equiv \inf M(x) .
\end{aligned}
$$

We will now continue strictly according to the proof of Theorem 3 in [2], pp. 242-243. For abbreviation set $s=\inf M(x)$. One can show that in view of the continuity of $v(p)$ and of (P4) there exists $\tilde{p} \in \mathrm{IR}_{++}^{n}$ such that $v(\tilde{p})=s$. Constructing a sequence

$s^{k}=s+\frac{1}{k}, \forall k \geq 1$, it follows that for every $s^{k}$ there exists $p^{k}$ such that $p^{k} x=1$ and $s \leq v\left(p^{k}\right)<s^{k}$. Since $\left\langle p^{k}\right\rangle$ is bounded there exists a convergent subsequence of $\left\langle p^{k}\right\rangle$, denoted by $\left\langle p^{k_{j}}\right\rangle$ such that $\lim _{j \rightarrow \infty} p^{k_{j}}=p^{0}$, and $s \leq \lim _{j \rightarrow \infty} v\left(p^{k_{j}}\right) \leq \lim _{j \rightarrow \infty} s^{k_{j}}=s<v\left(\frac{\tilde{p}}{2}\right)$. Hence, there does not exist a positive integer $N$ such that $p^{k_{j}} \succ \frac{\tilde{p}}{2}$ for all $j \geq N$. Application of (P5) to this result implies $p^{0}>0$. In view of the continuity of $v$ we obtain $\lim _{j \rightarrow \infty} v\left(p^{k_{j}}\right)=v\left(p^{0}\right)$, and therefore $v\left(p^{0}\right)=s$. Since $s=\inf M(x)$ and since $p^{0} x=1$, we have $p^{0} \preceq p^{\prime}, \forall p^{\prime} \in \mathrm{IR}_{++}^{n}$ such that $p^{\prime} x=1$. Therefore

${ }^{3}$ The (income normalized) indirect utility function $v$ is called decreasing if $p>p^{\prime} \Rightarrow v(p)<v\left(p^{\prime}\right)$, for all $p, p^{\prime} \in \mathrm{IR}_{++}^{n}$. $p^{0} \in\left\{p \in \mathrm{IR}_{++}^{n} \mid p \in B(x) \wedge \forall p^{\prime} \in B(x): v\left(p^{\prime}\right) \geq v(p)\right\}$.

Hence, $b(x) \neq \varnothing$ and thus $b$ can be well defined for all $x \in \mathrm{IR}_{++}^{n}$.

This concludes our proof.

If the inverse demand function is based on an indirect utility function satisfying the following condition ([2], p. 240):

$(\gamma)$ for every sequence

$\left\langle p^{k}\right\rangle, p^{k} \in \mathrm{IR}_{++}^{n},\left[p^{k} \rightarrow p^{0} \ngtr 0\right] \Rightarrow\left[v\left(p^{k}\right) \rightarrow \infty\right]$, then (P5) follows. Hence, $(\gamma)$ is stronger than (P5).

Lemma 2 Consider a continuous indirect utility function $v: \mathrm{IR}_{++}^{n} \rightarrow \mathrm{IR}_{+}$satisfying condition $(\gamma)$. Then the corresponding preference relation $\succeq$ defined by $p \succeq p^{\prime}: \Leftrightarrow v(p) \geq v\left(p^{\prime}\right)$, satisfies (P5).

Proof.

Given a sequence of price vectors $\left\langle p^{k}\right\rangle, p^{k} \in \mathrm{IR}_{++}^{n}$, such that $\lim _{k \rightarrow \infty} p^{k}=p^{0} \ngtr 0$, and a $p \in \operatorname{IR}_{++}^{n}$. By $(\gamma)$ it follows $v\left(p^{k}\right) \rightarrow \infty$. Since $v(p) \in \mathrm{IR}_{+}$and since $v\left(p^{k}\right) \rightarrow \infty$, there exsists a positive integer $N$ such that for all $k>N, v\left(p^{k}\right)>v(p)$, and hence $p^{k} \succ p$, concluding our proof.

According to (P5) the indirect utility function $v$ may converge to a finite value when $p^{k} \rightarrow p^{0} \ngtr 0$. Therefore, condition (P5) is weaker than $(\gamma)$.

\section{Some Properties of the Inverse Demand Correspondence}

It will now be shown that $b: \mathrm{IR}_{++}^{n} \rightarrow 2^{R_{++}^{n}}$ is upper hemicontinuous. We will characterize upper hemicontinuity by sequences ([6], pp. 262-263, Theorem A III. 1, part (b) of the proof, where compact-valuedness of the correspondence is not needed, see also [7], p. 532, Theorem 16.17):

Let $F: S \rightarrow 2^{T}$, where $S, T \subseteq \mathbb{I R}^{n}$, then $F$ is called "upper hemicontinuous" at $x^{0} \in S$, if for every sequence $\left\langle x^{k}\right\rangle$ with $\lim _{k \rightarrow \infty} x^{k}=x^{0}$ and for every sequence $\left\langle y^{k}\right\rangle$ with $y^{k} \in F\left(x^{k}\right)$, there exists a convergent subsequence of $\left\langle y^{k}\right\rangle,\left\langle y^{k j}\right\rangle$, such that $\lim _{j \rightarrow \infty} y^{k j}=y \in F\left(x^{0}\right) . F$ is called upper hemicontinuous if it is upper hemicontinuous at every $x \in S$.

Theorem 3 If the relation $\succeq$ satisfies $(P 1)$ to $(P 5)$ and if $b: \mathbb{R}_{++}^{n} \rightarrow 2^{R_{++}^{n}}$ is consistent with $\succeq$, then $b$ is upper hemicontinuous.

\section{Proof.}

Let $\left\langle x^{k}\right\rangle, x^{k} \in \mathrm{IR}_{++}^{n}$, be a sequence such that $\lim x^{k}=\tilde{x} \in \operatorname{IR}_{++}^{n}$. Thus, for any $k$ and for all $p^{\prime} \in \mathbb{I R}_{++}^{n}$ such that $p^{\prime} x^{k} \leq 1$ it follows, $p^{\prime} \succeq p^{k}$, where 
$p^{k} \in b\left(x^{k}\right)$ and $p^{k}=\left(p_{1}^{k}, \cdots, p_{n}^{k}\right)$. If $\left\langle p^{k}\right\rangle$ is not bounded, then there must exist a positive integer $j \leq n$ and a sequence $\left\langle p_{j}^{k}\right\rangle$ such that $\lim _{j \rightarrow \infty} p_{j}^{k}=\infty$. Since

$\lim _{k \rightarrow \infty} x_{j}^{k}=\tilde{x}_{j}>0$, we obtain $p_{l}^{k} x_{l}^{k}>1$ for some $m \in \mathbb{I N}$ and for all $l>m$, contradicting $p^{k} x^{k} \leq 1$. Hence $\left\langle p^{k}\right\rangle$ is bounded, and therefore, taking a subsequence if necessary, $\lim _{k \rightarrow \infty} p^{k}=\tilde{p}$. In order to obtain a contradiction, suppose $\tilde{p} \ngtr 0$. Obviously, there exists $\bar{p} \in \mathrm{IR}_{++}^{n}$ such that $\bar{p} \cdot \tilde{x}<1$. Since $\lim _{k \rightarrow \infty} x^{k}=\tilde{x}$ there also exists an integer $N^{1}>0$ such that for all $k>N^{1}: \bar{p} \cdot x^{k}<1$. Hence, by consistency, $p^{k} \preceq \bar{p}$. On the other side, (P5) yields the existence of an integer $N^{2}>0$ such that for all $k>N^{2}, p^{k} \succ \bar{p}$. Take $\tilde{N}=\max \left\{N^{1}, N^{2}\right\}$, then for all $k>\tilde{N}, \quad p^{k} \succ \bar{p}$ and $p^{k} \preceq \bar{p}$, a contradiction. Hence, $\tilde{p}>0$.

Now, it will be shown that $\tilde{p} \in b(\tilde{x})$. Therefore, let us consider $\hat{p} \in \operatorname{IR}_{++}^{n}$ such that $\hat{p} \tilde{x} \leq 1$. In order to obtain that $\tilde{p} \preceq \hat{p}$, we have to consider two cases.

1) $\hat{p} \tilde{x}<1$.

Hence, there exists a positive integer $N$ such that $\hat{p} x^{k}<1$ for all $k>N$, and therefore $p^{k} \preceq \hat{p}$, for all $k>N$. In view of lower semicontinuity of $\preceq$,

$\lim _{k \rightarrow \infty} p^{k}=\tilde{p} \preceq \hat{p}$.

2) $\hat{p} \tilde{x}=1$.

Let $\left\langle q^{l}\right\rangle, q^{l} \in \mathrm{IR}_{++}^{n}$, be a sequence such that

$\lim _{k \rightarrow \infty} q^{l}=\hat{p}$ and $q^{l} \tilde{x}<1$. Obviously, such a sequence does exist. Since we have $q^{l} x^{k}<1$ for some positive integer $\hat{N}$ and for all $k>\hat{N}$, we obtain $p^{k} \preceq q^{l}$. By lower semicontinuity this implies, $\lim _{k \rightarrow \infty} p^{k}=\tilde{p} \preceq q^{l}$. In view of upper semicontinuity of $\preceq$, we thus obtain $\tilde{p} \preceq \hat{p}$. Hence $\tilde{p} \in b(\tilde{x})$. This concludes our proof

Corollary: In the light of Lemma 2 and the previous remarks it follows: given a continuous and decreasing indirect utility function $v: \mathrm{IR}_{++}^{n} \rightarrow \mathrm{IR}_{+}$satisfying condition $(\gamma)$, then the inverse demand correspondence $b$ : $\mathrm{IR}_{++}^{n} \rightarrow 2^{\mathbb{R}_{++}^{n}}$, defined by

$b(x)=\left\{p \in B(x) \mid v(p) \leq v\left(p^{\prime}\right)\right.$, for all $\left.p^{\prime} \in B(x)\right\}$ is upper hemicontinuous.

Finally, since strict concavity of $\succeq$ implies singlevaluedness of the inverse demand correspondence we immediately obtain the following theorem. Preliminarily, remember that strict concavity is defined as follows: let
$Y$ be a convex set, and let $p, p^{\prime} \in Y$ with $p \neq p^{\prime}$, then:

$$
p \succeq p^{\prime} \Rightarrow p \succ \lambda p+(1-\lambda) p^{\prime}, \forall \lambda \in(0,1) .
$$

Theorem 4 Let $\succeq$ satisfy $(P 1)$ to $(P 5)$ and let it be strictly concave, then

a) $b(x)$ is single-valued if $b$ is consistent with $\succeq$, and $b: \mathrm{IR}_{++}^{n} \rightarrow \mathrm{IR}_{++}^{n}$ is a continuous function,

b) if $\succeq$ is homothetic ${ }^{4}$, then $b$ is homogeneous of degree -1 .

Note, that upper hemicontinuity and continuity are identical, when $b$ is a function ([6], p. 262).

\section{Summary}

In the previous analysis, a formal frame for modelling consumer behavior has been presented when firms offer certain amounts of commodities at the market and consumers react to these offers by their willingness to pay. This willingness to pay can be described by the inverse demand correspondence. When the plans of consumers and firms coincide then the deal will take place, and the consumers will achieve the goods.

\section{REFERENCES}

[1] A. Mas-Colell, M. D. Whinston and J. R. Green, "Microeconomic Theory, Volume 1," Oxford University Press, New York, 1995.

[2] G. Debreu, "Theory of Value: An Axiomatic Analysis of Economic Equilibrium," Yale University Press, New Haven, 1959.

[3] S. Fuchs-Seliger, "A Note on Duality in Consumer Theory," Economic Theory, Vol. 13, No. 1, 1999, pp. 239246. doi: $10.1007 / \mathrm{s} 001990050252$

[4] W. Erwin Diewert, "Applications of Duality Theory," In: M. D. Intriligator and D. A. Kendrick, Eds., Frontiers of Quantitative Economics, Vol. II, North-Holland, Amsterdam, 1974, pp. 106-176.

[5] S. Fuchs-Seliger, "Compensated and Direct Demand without Transitive and Complete Preferences," Annals of Operations Research, Vol. 23, No. 1, 1990, pp. 299-310. doi:10.1007/BF02204854

[6] C. D. Aliprantis and C. Kim, "Infinite Dimensional Analysis: Hitchhikers Guide," Springer-Verlag, New York, 1986.

[7] W. Hildenbrand and A. P Kirman, "Equilibrium Analysis," North-Holland Publishing Company, Amsterdam, 1988.

\footnotetext{
${ }^{4} \succeq$ is called "homothetic", if $x \succeq y \rightarrow \lambda x \succeq \lambda y, \forall \lambda>0$.
} 